Reprod. Nutr. Dévelop., 1982, 22 (6), 941-949.

\title{
Influence d'une élévation de la température de l'eau sur la digestibilité de la matière sèche, de l'azote et de l'énergie de l'aliment distribué à la truite arc-en-ciel (Salmo gairdneri Rich)
}

\author{
G. CHOUBERT, B. FAUCONNEAU, P. LUQUeT
}

avec la collaboration technique de Denise BLANC et Jeannine BREQUE

Laboratoire de Nutrition et d'Elevage des poissons, I.N.R.A. Saint-Pée-sur-Nivelle, 64310 Ascain, France

Summary. Influence of a rise in water temperature on food dry matter, nitrogen and energy digestibility of rainbow trout (Salmo gairdneri Rich.).

Rainbow trout adapted to a water temperature of $10{ }^{\circ} \mathrm{C}$ were subjected to an abrupt rise in temperature (from 10 to $18{ }^{\circ} \mathrm{C}$ ) in a 24-h period. Fish maintained in recirculated water were fed to satiation twice a day and their feed intakes were recorded. Changes in dry matter, nitrogen and energy digestibility were measured each day at $10^{\circ} \mathrm{C}$ and during the course of acclimatation to $18^{\circ} \mathrm{C}$. Low water temperature $\left(10^{\circ} \mathrm{C}\right)$ was characterized by a feed intake of $1.84 \mathrm{~g}(\mathrm{DM}) /$ fish/day; digestibility values were as follows : dry matter 62.15 p. 100 , nitrogen 86.91 p. 100 , energy 70.60 p. 100 . High water temperature $\left(18{ }^{\circ} \mathrm{C}\right)$ was characterized by a feed intake of $3.75 \mathrm{~g}(\mathrm{DM}) / \mathrm{fish} /$ day; digestibility values were as follows : dry matter 66.08 p. 100 , nitrogen 89.57 p. 100 , energy 73.52 p. 100 . The daily patterns in digestibility were affected by the rise in temperature. The digestibility values were stabilized by day 7 after the positive thermal shock.

\section{Introduction.}

Poecilothermes, les poissons sont sensibles aux variations de température de leur environnement et réalisent des ajustements notamment au niveau digestif. Ainsi, l'ensemble des processus digestifs (transit, digestion, absorption) semble stimulé par une augmentation transitoire ou prolongée de température. Toutefois, les conséquences sur le bilan digestif, exprimées par le coefficient d'utilisation digestive semblent variables. Les différents travaux réalisés jusqu'à l'heure ont porté essentiellement sur l'étude des poissons acclimatés à différentes températures.

Karzinkin (1935) chez la carpe, Cyprinus carpio, de même qu'Arnoldi et Fortunatova (1937) chez le gobie, Gobius cephalarges, rapportent une amélioration de 18 p. 100 du coefficient d'utilisation digestive (CUD) de la matière sèche de l'aliment lorsque la température de l'eau des bassins est augmentée de 10 à $13^{\circ} \mathrm{C}$. 
Par contre, Windell et al. (1978) puis Cho et Slinger (1979) ne notent pas de différence de digestibilité de la matière sèche chez les truites arc-en-ciel, Salmo gairdneri, acclimatées à $7{ }^{\circ} \mathrm{C}$ ou à $15^{\circ} \mathrm{C}$.

L'influence du facteur thermique sur la digestibilité des protéines n'a, semble-t-il, pas pu être mise en évidence chez le mérou, Epinephelemus guttatus (Menzel, 1960), l'écrevisse, Procambarus clarkii (Nose, 1964) et la perche, Stizostedion vitreum (Kelso, 1972). Chez la truite arc-en-ciel, si Possompes (1973) n'a pas observé de variations de la digestibilité des protéines avec la température d'acclimatation, Windell et al. (1978) et Cho et Slinger (1979) mentionnent une digestibilité de l'azote plus élevée aux températures de 15 à $18{ }^{\circ} \mathrm{C}$ qu'à celles de 7 à $9{ }^{\circ} \mathrm{C}$, cette différence atteignant 1 à 2 points.

Pour l'énergie, Andrews et al. (1978) notent une différence de digestibilité de 20 à 30 p. 100 chez le poisson chat, Ictalurus punctatus, élevé à des températures de 22 à $28{ }^{\circ} \mathrm{C}$, tandis que Windell et al. (1978) ne trouvent aucune différence chez des truites arc-en-ciel acclimatées à $7^{\circ}$ ou $15^{\circ} \mathrm{C}$.

Ces différents résultats, apparemment contradictoires, $n^{\prime}$ ont qu'une valeur comparative restreinte et ne permettent pas de conclure. L'étude de la digestibilité après une élévation thermique peut apporter des informations supplémentaires d'une part sur les variations de la digestibilité avec la température d'acclimatation et d'autre part sur la mise en place d'un nouvel équilibre dans les processus digestifs en mettant en évidence l'existence ou non d'une phase transitoire au cours de laquelle le bilan digestif est altéré. Ainsi, au cours de la présente étude qui s'inscrit dans le cadre plus général de l'étude des facteurs de variations du transit et de la digestibilité chez les poissons, nous nous sommes attachés à définir les conséquences d'une élévation de température sur la digestibilité de la matière sèche, de l'azote et de l'énergie dans des conditions expérimentales bien précises.

\section{Matériel et méthodes.}

L'évolution des CUD est suivie journellement avant et après une élévation thermique de $8{ }^{\circ} \mathrm{C}$.

a) Conditions d'élevage. - L'expérience a été réalisée sur des truites arcen-ciel, Salmo gairdneri Rich., d'un poids initial de $160 \mathrm{~g}$ réparties au hasard en 6 lots de 15 individus, placés dans des bacs cylindroconiques de $40 \mathrm{~cm}$ de diamètre et $d^{\prime}$ une capacité utile de 50 litres. Les bacs expérimentaux, installés sous abris, reçoivent un éclairage artificiel pendant $12 \mathrm{~h}$ par jour et sont alimentés en parallèle par un circuit d'eau semi-fermé assurant un débit de l'ordre de $4 \mathrm{l} / \mathrm{min} /$ bac. La thermorégulation est assurée par une source froide d'une puissance de 20000 frigories $/ \mathrm{h}$ et une source chaude d'une puissance de $22000 \mathrm{kcal} / \mathrm{h}$.

Les truites reçoivent chaque jour deux repas à $8 \mathrm{~h} 30$ et à $16 \mathrm{~h} 30$. L'aliment dont la composition est donnée dans le tableau 1 est distribué à chaque repas selon la méthode décrite par Luquet (1971).

b) Régime thermique. - Préalablement à l'expérience les poissons sont acclimatés pendant un mois à une température de $10^{\circ} \mathrm{C} \pm 0,5^{\circ} \mathrm{C}$. La tempéra- 
TABLEAU 1

Composition centésimale de l'aliment expérimental. Analyse du régime

\begin{tabular}{|c|c|}
\hline & (p. 100) \\
\hline 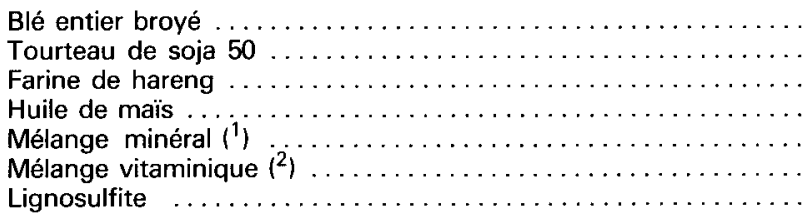 & $\begin{array}{r}33 \\
22 \\
35 \\
5 \\
1 \\
2 \\
2\end{array}$ \\
\hline 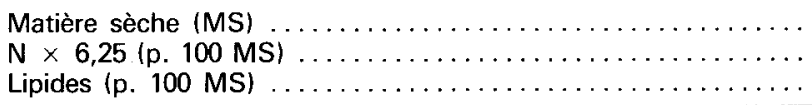 & $\begin{array}{r}91,51 \\
8,53 \\
40,58\end{array}$ \\
\hline
\end{tabular}

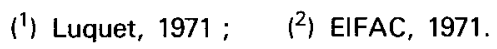

ture de l'eau est ensuite augmentée en une journée $\left(1^{\circ} \mathrm{C} / \mathrm{h}\right)$ jusqu'à $18^{\circ} \mathrm{C} \pm$ $0,5^{\circ} \mathrm{C}$.

c) Méthode et séquence de prélèvement. - La récolte des fèces s'effectue à l'aide du dispositif précédemment décrit (Choubert et al., 1979) dont le principe est le suivant : l'eau d'évacuation des aquariums qui contiennent les poissons passe à travers des grilles métalliques qui retiennent les fèces, les séparent de l'eau et par un mouvement de rotation les soustraient du lessivage et les projettent sur des plateaux où elles sont immédiatement congelées.

Les digestibilités de la matière sèche, de l'azote et de l'énergie sont déterminées journellement : à $10{ }^{\circ} \mathrm{C}$ pendant une période de 6 jours au terme de la période d'acclimatation à cette température; à $18{ }^{\circ} \mathrm{C}$ pendant les 8 premiers jours puis les jours 13 et 14, 20 et 21,27 à 29 après l'augmentation de température.

d) Méthode d'analyse et de calcul. - Chaque jour, les plateaux contenant les fèces sont changés et leur contenu est lyophilisé, broyé puis conservé en piluliers hermétiques jusqu'aux analyses. Les méthodes d'analyses et de calcul ont été décrites par de la Nouë et al. (1980).

\section{Résultats.}

a) Influence de la température de l'eau. - Les valeurs moyennes journalières des consommations d'aliment d'une part et des digestibilités de la matière sèche, de l'azote et de l'énergie d'autre part, sont rapportées dans la figure 1.

A basse température $\left(10^{\circ} \mathrm{C}\right)$ pendant une période de 6 jours, la consommation moyenne est de $1,84 \pm 0,29 \mathrm{~g}$ d'aliment (MS) par poisson et par jour. Pendant la même période, les moyennes générales des valeurs des digestibilités quotidiennes de la matière sèche, de l'azote et de l'énergie sont respectivement $62,15 \pm 0,60$ p. $100,86,91 \pm 0,74$ p. 100 et $70,60 \pm 1,09$ p. 100 . Les valeurs des moyennes journalières se trouvent dans l'intervalle de confiance de la moyenne générale tant pour la matière sèche que pour l'azote et l'énergie.

A haute température $\left(18^{\circ} \mathrm{C}\right)$, la consommation d'aliment est deux fois plus élevée $(3,75 \pm 0,26 \mathrm{~g} \mathrm{MS} /$ poisson/jour) qu'à basse température. Les valeurs 


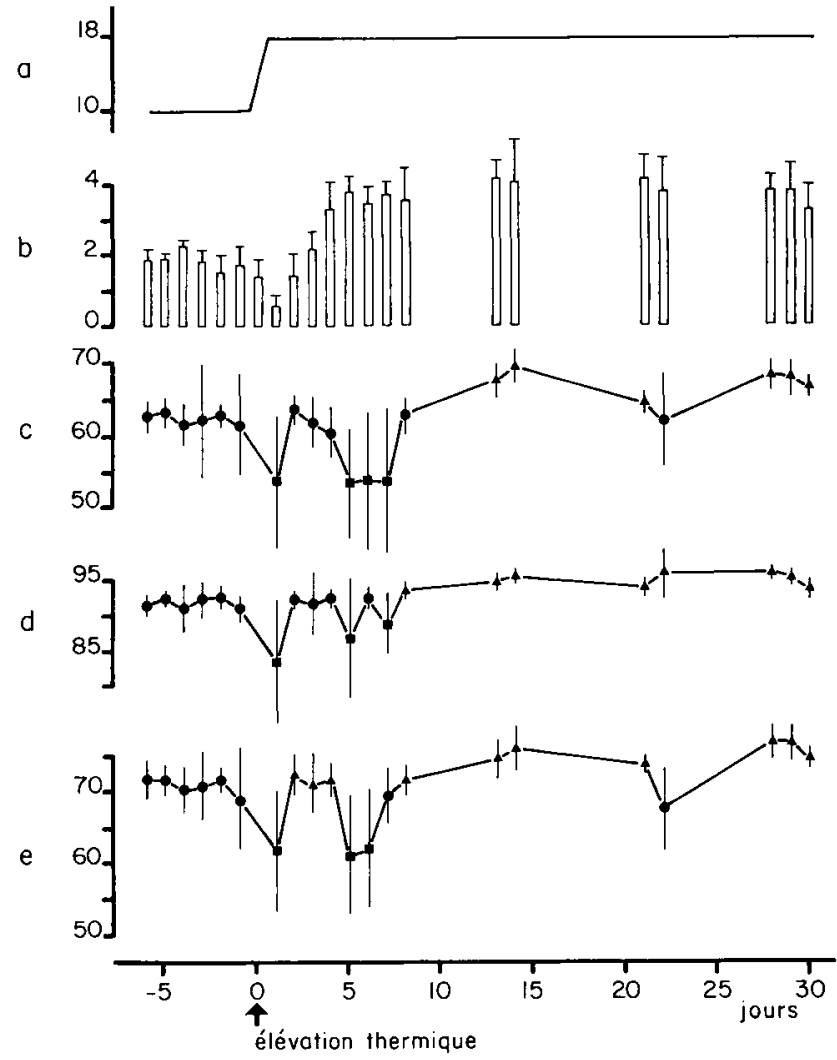

FIG. 1. - Evolution journalière des consommations d'aliment par le poisson et des digestibilités de la matière sèche, de l'azote et de l'énergie aux températures de $10^{\circ} \mathrm{C}$ et $18^{\circ} \mathrm{C}$.

$\mathrm{a}=$ évolution de la température de $10^{\circ} \mathrm{C}$ à $18^{\circ} \mathrm{C} ; \mathrm{b}=$ consommation d'aliment par poisson (g matière sèche/poisson/jour); $\mathrm{c}=$ coefficient $\mathrm{d}^{\prime} u$ tilisation digestive de la matière sèche (p. 100) ; $d=$ coefficient d'utilisation digestive de l'azote ( $p$. 100 matière sèche) ; $\mathbf{e}=$ coefficient $\mathrm{d}^{\prime} u$ tilisation digestive de l'énergie ( $p$. 100 matière sèche).

Sur les figures $c, d, e$, deux valeurs de coefficient d'utilisation digestive, affectées de symboles différents sont significativement différentes.

des CUD sont également plus élevées qu'à $10^{\circ} \mathrm{C}$. Ainsi, pendant une période de 11 jours (du jour 8 au jour 29), les moyennes générales des CUD sont égales à $66,08 \pm 2,64$ p. 100 pour la matière sèche, $89,57 \pm 0,94$ p. 100 pour l'azote et $73,52 \pm 3,02$ p. 100 pour l'énergie. Les moyennes journalières des CUD de la matière sèche, de l'azote et de l'énergie se situent également dans l'intervalle de confiance de la moyenne générale.

En effectuant le test statistique $t$ de Student (tabl. 2) entre les moyennes des valeurs des CUD à haute et basse température, il apparaît une différence hautement significative (seuil de 1 p. 100) pour les CUD de la matière sèche et de l'azote. Pour les CUD de l'énergie, cette différence n'est significative qu'au seuil de 5 p. 100. 
TABLEAU 2

Comparaison des moyennes générales des valeurs des CUD de la matière sèche de l'azote et de l'énergie aux températures de $10^{\circ} \pm 1{ }^{\circ} \mathrm{C}$ et $18^{\circ} \pm 1{ }^{\circ} \mathrm{C}$

\begin{tabular}{|c|c|c|c|}
\hline & $\begin{array}{c}\text { CUD } \\
\text { matière sèche } \\
(p .100)\end{array}$ & $\begin{array}{c}\text { CUD } \\
\text { azote } \\
\text { (p. 100) }\end{array}$ & $\begin{array}{c}\text { CUD } \\
\text { énergie } \\
\text { (p. 100) }\end{array}$ \\
\hline $\begin{array}{l}\text { Moyenne générale } \\
\text { à } 10^{\circ} \mathrm{C} \pm 1{ }^{\circ} \mathrm{C} \\
\text { pour j } 6 \text { à j } 1 \ldots \ldots\end{array}$ & 62,15 & 86,91 & 70,60 \\
\hline Déviation standard & 0,65 & 0,74 & 1,09 \\
\hline $\begin{array}{l}\text { Moyenne générale } \\
\text { à } 18^{\circ} \mathrm{C} \pm 1{ }^{\circ} \mathrm{C} \\
\text { pour j } 8 \text { à j } 29 \ldots\end{array}$ & 66,08 & 89,57 & 79,52 \\
\hline Déviation standard & 2,64 & 0,94 & 3,02 \\
\hline $\begin{array}{l}\text { Test } t \text { de Student } \\
\mathrm{t} \text { calculé } \ldots \ldots \ldots \\
\mathrm{t} \text { théorique } \ldots \ldots \ldots \\
\text { Différence } \ldots \ldots \ldots\end{array}$ & $\begin{array}{r}3,62 \\
3,06 \\
H S^{*}\end{array}$ & $\begin{array}{l}5,70 \\
3,06 \\
\text { HS }\end{array}$ & $\begin{array}{l}2,24 \\
2,20 \\
S^{* *}\end{array}$ \\
\hline
\end{tabular}

${ }^{*} \mathrm{H} \mathrm{S}=$ Hautement significatif $;{ }^{*} \mathrm{~S}=$ Significatif.

b) Influence de l'élévation de température de l'eau. Les valeurs des CUD de la matière sèche, de l'azote et de l'énergie sont très irrégulières après le changement de température de l'eau pendant une période d'environ 7 jours. Deux diminutions brutales des CUD d'environ 10 p. 100 ont été notées aux jours 1 et 5 .

Afin de définir le jour à partir duquel on peut considérer que les valeurs des moyennes journalières des CUD sont stabilisées, la plus petite différence significative (PPDS) a été calculée selon Snedecor et Cohran (1971). Sur la figure 1, la PPDS a été représentée en indexant de façon distincte les valeurs moyennes des CUD significativement différentes.

La stabilisation des valeurs des CUD de la matière sèche à $18{ }^{\circ} \mathrm{C}$ n'est réalisée que le $14^{e}$ jour après l'élévation de la température $(69,5 \pm 2,6$ p. 100).

\section{Discussion.}

Influence de la température d'acclimatation. - L'eftet de la température de l'environnement se manifeste chez la truite arc-en-ciel, par l'augmentation des quantités alimentaires ingérées d'une part et par l'accroissement de la vitesse de transit d'autre part.

La consommation alimentaire des poissons, en réponse à l'élévation de température de l'eau, augmente rapidement après une phase transitoire. En effet, la consommation chute brusquement d'environ 60 p. 100 le premier jour et se rétablit les jours suivants. La simultanéité de deux phénomènes distincts pourrait expliquer cette observation : d'une part un manque d'oxygène dans l'eau, occasionné par l'élévation de température (Kaushik, 1981) et d'autre part un besoin accru des poissons en aliment à cette nouvelle température (Rozin et Mayer, 1961 ; Baldwin, 1956). Lorsque la teneur en oxygène est rétablie par bullage d'air comprimé dans l'eau, l'accroissement du besoin alimentaire n'est pas immédiat 
mais s'échelonne sur une période de temps de 4 jours après l'élévation de température pour se stabiliser par la suite. Cette observation est tout à fait comparable à celle rapportée antérieurement par Fauconneau et Luquet (1979) et Kaushik (1981).

S'il est généralement admis que la vitesse de transit suit la loi d'Arrhenius (Molnar et Tölg, 1962 ; Shrabble et al., 1969 ; Brett et Higgs, 1970 ; Edwards, 1971 ; Elliott, 1972 ; Possompes, 1973 ; Windell et al., 1976 ; Jobling et al., 1977 ; Ross et Jauncey, 1981), l'augmentation de la vitesse de transit alimentaire avec la température devrait entraîner un abaissement de la digestibilité par diminution du temps de contact enzyme digestive-substrat (Windell et al., 1978 ; Luquet et Fauconneau, 1979 ; Cho et Slinger 1979). Nos observations vont à l'encontre de cette hypothèse; à la température de $18{ }^{\circ} \mathrm{C}$, les digestibilités de la matière sèche, de l'azote et de l'énergie sont supérieures de 3 à 4 points à celles observées à $10{ }^{\circ} \mathrm{C}$. Ces résultats corroborent ceux d'Arnoldi et Fortunatova (1937) et de Karzinkin (1935). Il existerait donc un phénomène de compensation de la capacité digestive en réponse à une augmentation de la vitesse de transit. Selon l'hypothèse formulée par Hochachka (1967), ce phénomène comprendrait le contrôle de la synthèse de l'enzyme d'une part, et de la fonction de l'enzyme d'autre part, mais également induirait une modification de l'affinité entre l'enzyme et le substrat (Somero, 1969 ; Léger et al., 1976).

Influence de l'augmentation de la température de l'eau. - L'évolution des coefficients d'utilisation digestive indique que l'ensemble des réajustements semble réalisé le $8^{\mathrm{e}}$ jour après l'élévation de température de l'eau. On peut donc estimer que l'adaptation des phénomènes digestifs est réalisée au bout d'une semaine.

Durant les premiers jours après l'élévation de la température de l'eau on constate deux phénomènes : une diminution brutale de la digestibilité le premier jour suivie d'une augmentation transitoire puis une seconde réduction durant les jours $5,6,7$. On peut signaler que les diminutions de digestibilité s'accompagnent d'une très grande variabilité de l'écart type observé sur la digestibilité. La réduction de la digestibilité, le premier jour de changement de température, pourrait s'expliquer indirectement par la réduction brutale de la quantité d'aliment ingérée. II peut s'agir également d'une réaction consécutive au stress thermique soit par ses effets primaires, soit par ses effets secondaires (Mazeaud et al., 1977). Toutefois dans nos conditions expérimentales, le choc thermique ne devrait pas exister car l'élévation de température est lente $\left(1^{\circ} \mathrm{C} / \mathrm{h}\right)$ et demeure proche des conditions naturelles. Pourtant, même dans les conditions naturelles, le choc thermique a été observé (Boehlke et al., 1966 ; Singley et Chavin, 1971 ; Srivastava et Meier, 1972 ; Garcia et Meier, 1973 ; Meier et Srivastava, 1975). Cet effet de choc disparaîtrait le $2^{\circ}$ jour et la valeur de la digestibilité ne serait plus que la résultante de deux effets contraires : augmentation de la vitesse de transit et stimulation des activités enzymatiques (Owen et Wiggs, 1971). On note également que la consommation d'aliment dès le $2^{\mathrm{e}}$ jour atteint le niveau qu'elle avait avant l'élévation de la température de l'eau. Ainsi, les stimulations des phénomènes digestifs et de l'absorption sont déjà mises en place dès le $2^{e}$ jour après l'élévation de température de l'eau. 
Le $5^{e}$ et le $6^{e}$ jour après l'élévation thermique apparaît une nouvelle diminution brutale de digestibilité de la matière sèche, de l'azote et de l'énergie. Si l'effet de l'augmentation de la consommation ne peut être mis en cause ici, il convient donc d'admettre que les mécanismes digestifs mis en place sont différents de ceux observés au $2^{e}$ jour. En effet, la digestibilité n'étant, en fait, qu'un bilan, la stimulation de ses différentes composantes par la température ne fait pas appel aux mêmes mécanismes car elles n'ont pas toutes le même $Q$ 10. Ces hypothèses explicatives méritent toutefois d'être vérifiées.

Après le $8^{\mathrm{e}}$ jour, les valeurs de digestibilité et de consommation sont stabilisées, indiquant que le poisson est acclimaté à son nouvel environnement. Ce résultat rejoint celui de Luquet et Fauconneau (1979) et confirme qu'une période d'acclimatation plus longue (Demael et Perez, 1974 ; Hazel et Prosser, 1974) semble excessive. Toutefois on peut signaler qu'il existe une variabilité individuelle au niveau des valeurs de CUD même lorsque, les conditions expérimentales semblent constantes. Des écarts types plus faibles et une précision relative de l'ordre de 1 à 3 p. 100 ne pourraient être obtenus qu'avec des dispositifs expérimentaux beaucoup plus importants (de la Nouë et al., 1980).

En conséquence, en maîtrisant l'augmentation de la température de l'eau et en réduisant l'effet de choc, on peut induire, chez la truite arc-en-ciel, un bilan digestif supérieur ou identique à température élevée qu'à basse température, pour une consommation alimentaire double ; ce qui se traduira par une quantité de nutriment disponible nettement supérieure.

Reçu en mars 1982.

Accepté en juin 1982.

Remerciements. - Les auteurs remercient Monsieur S. Kaushik pour son active collaboration.

\section{Références}

ANDREWS J. W., MURRAY M. W., DAVIS J. M., 1978. The influence of dietary fat dietary fat levels and environmental temperature on digestible energy and absorbability of animal fat in catfish diet. J. Nutr., 108, 749-752.

ARNOLDI L. V., FORTUNATOVA K. R., 1937. On the experimental studying of fish feeding in the black sea. Dokl. Akad. Nauk. SSSR, 15, 505-508.

BALDWIN N. S., 1956. Food consumption and growth of brook trout at different temperature. Trans. am. Fish. Soc., 86, 323-328.

BOEHKLE K. W., CHURCH R. L., TIEMEIER O. W., ELEFTHERIOU B. E., 1966. Diurnal rhythm in plasma glucocorticoid levels in channel catfish (/ctalurus punctatus). Gen. comp. Endocrinol., 7, 18-21.

BRETT J. R., HIGGS D. A., 1970. Effects of temperature on the rate of gastric digestion in fingerling sockeye salmon Oncorhynchus nerka. J. Fish. Res. Bd Can., 27, 1767-1779.

CHO C. Y., SLINGER S. J., 1979. Apparent digestibility measurement in feedstuffs for rainbow trout. In HALVER J. E., TIEWS K., Proc. World Symp. on Finfish nutrition and fishfeed technology, Hamburg, 20-23 June 1978, Vol. II, 239-247.

CHOUBERT G., De la NOÜE J., LUQUET P., 1979. Continuous quantitative automatic collector for fish feces. Progr. Fish-Cult., 41, 64-67. 
De la NOÜE J., ChOUBERT G., PAGNIEZ B., BLANC J. M., LUQUeT P., 1980. Digestibilité chez la truite arc-en-ciel (Salmo gairdneri) lors de l'adaptation à un nouveau régime alimentaire. Can. J. Fish. Aquat. Sci., 37, 2218-2224.

DEMAEL A., PEREZ G., 1974. Influence de la température sur le métabolisme des poissons. Cah. Lab. Hydrobiol. Montereau, 1, 21-26.

EDWARDS D. J., 1971. Effect of temperature on rate of passage of food through the alimentary canal of the plaice Pleuronectes platessa L. J. Fish. Biol., 3, 433-439.

EIFAC, 1971. European Fisheries advisory Commission. Salmo and trout feeds and feeting. FAO Tech. Pap., Rome, 12, 29 pp.

ELLIOTT J. M., 1972. Rates of gastric evacuation in brown trout Salmo trutta L., Freshwat. Biol., 2. 1-18.

FAUCONNEAU B., LUQUET P., 1979. Influence d'une élévation de température sur l'évolution de l'aminoacidémie et de l'ammoniémie après le repas chez la truite arc-en-ciel (Salmo gairdneri R.). Ann. Biol. anim. Bioch. Biophys., 19, 1063-1079.

GARCIA L. E., MEIER A. H., 1973. Daily rhythms in concentrations of plasma cortisol in male and female gulf killifish Fundulus grandis. Biol. Bull., 144, 471-479.

HAZEL J. R., PROSSER C. L., 1974. Molecular mechanism of temperature compensation in poikilotherm. Physiol. Rev., 54, 620-671.

HOCHACHKA P. W., 1967. Organization of metabolism during temperature compensation, 117-203. In C. L. PROSSER. Molecular mechanisms of temperature adaptation. Am. Ass. Adv. Sci., Washington.

JOBLING M., GWYTHER D., GROVE D. J., 1977. Some effects of temperature meal size and body weight on gastric evacuation time in the dab Limanda limanda (L.). J. Fish. Biol., 10, 291-298.

KARZINKIN G. S., 1935. Zur kentniss der Fishproductivität der gewässer. II. Mitteilung. Erforschung der Physiologie der Ernährung des Spielgelkarpfens. Arb. Limnol. Sta. Kossino, 19, 21-66.

KAUSHIK S. J., 1981. Influence of a rise in temperature on the nitrogen excretion of rainbow trout (Salmo gairdneri R.). Proc. World Symp. on aquaculture in heated effluents and recirculation systems, Stavanger, 28-30 mai 1980, vol. 1, 77-89.

KELSO J. R. M., 1972. Conversion, maintenance and assimilation for walleye (Stizastedion vitreum vitreum) as affected by size, diet and temperature. J. Fish. Res. Bd Can., 29, 1181-1192.

LEEGER C., LUQUET P., BOUDON M., 1976. Effets de la suppression des glucides dans I'alimentation de la truite arc-en-ciel à la température de $16^{\circ} \mathrm{C}$. II. Evolution des compartiments corporels avec référence particulière aux acides gras des tamilles $\omega 9 \omega 6 \omega 3$ et influence d'un changement de température. Ann. Hydrobiol., 7, 185-201.

LUQUET P., 1971. Efficacité des protéines en relation avec leur taux d'incorporation dans l'alimentation de la truite arc-en-ciel. Ann. Hydrobiol., 2, 175-186.

LUQUET P., FAUCONNEAU B., 1979. Influence de la température sur quelques aspects de l'alimentation des poissons. Bull. Cent. Etud. Rech. sci., Biarritz, 12, 475-492.

MAZEAUD M. M., MAZEAUD F., DONALDSON E. M., 1977. Primary and secondary effects on stress in fish : Some new data with a general review. Trans. am. Fish. Soc., 106, 201-212.

MEIER A. H., SRIVASTAVA A. K., 1975. Alteration by drugs of the daily rhythm of plasma corticosteroid concentration in the gulf killifish Fundulus grandis. Gen. comp. Endocrinol., 26, 253-258.

MENZEL D. W., 1960. Utilization of food by a bermuda reef fish, Epinephalus guttatus. J. Cons. perm. int. Explor. Mer, 25, 216-222.

MOLNAR G., TÖLG I., 1962. Relation between water temperature and gastric digestion of large mouth bass (Micropterus salmoides Lacepede). J. Fish. Res. Bd Can., 19, 1005-1012.

NOSE T., 1964. Protein digestibility of several test diets in cray and prawn fish. Bull. Freshwat. Fish. Res. Lab. Tokyo, 14, 23-28.

OWEN T. G., WIGGS A. J., 1971. Thermal compensation in the stomach of the brook trout (Salvelinus fontinalis Mitchill). Comp. Biochem. Physiol., 40 b, 465-473.

POSSOMPES B., 1973. Influence de la température sur les besoins en protéines, le transit alimentaire et la digestibilité chez la truite arc-en-ciel (Salmo gairdneri Rich.). Th. $3^{e}$ cycle, Univ. Paris $6,58 \mathrm{pp}$. 
ROSS B., JAUNCEY K., 1981. A radiographic estimation of the effect of temperature on gastric emptying time in Sarotherodon niloticus $\mathrm{L} \times S$. aureus Steindachner hybrids. J. Fish. Biol., 19, 333-344.

ROZIN P., MAYER J., 1961. Some factors influencing short term food intake of the goldfish. Amer. J. Physiol., 206, 1430-1436.

SHRABBLE J. B., TIEMEIER O. W., DEYOE C. W., 1969. Effects of temperature on rate of digestion by channel catfish. Progr. Fish-Cult., 31, 131-138.

SINGLEY J. A., CHAVIN W., 1971. Cortisol levels of normal goldfish Carassius auratus L. and response to osmotic change. Am. Zool., 11, 653.

SNEDECOR G. W., COCHRAN W. G., 1967. Statistical methods. 6th ed. lowa State Univ. Press, Amos (USA), $535 \mathrm{pp}$.

SOMERO G. N., 1969. Enzymic mechanisms of temperature compensation : Immediate and evolutionary effects of temperature on enzymes of aquatic poikilotherms. Amer. Nat., 103, 517-530.

SRIVASTAVA A. K., MEIER A. H., 1972. Daily variation in concentration of cortisol in plasma in intact and hypophysectomized gulf killifish. Science, 177, 185-187.

WINDELL J. T., FOLTZ J. W., SAROKON J. A., 1978. Effect of fish size temperature and amount fed on nutrient digestibility of a pelleted diet by rainbow trout Salmo gairdneri. Trans. am. Fish. Soc., 107, 613-616.

WINDELL J. T., KITCHELL J. F., NORRIS D. D., NORRIS J. S., FOLTZ J. W., 1976. Temperature and rate of gastric evacuation by rainbow trout (Salmo gairdneri). Trans. am. Fish. Soc., 6, 712-717. 